\title{
Neonatal outcomes of moderately preterm infants compared to extremely preterm infants
}

\author{
Michele C. Walsh ${ }^{1}$, Edward F. Bell ${ }^{2}$, Sarah Kandefer ${ }^{3}$, Shampa Saha $^{3}$, Waldemar A. Carlo ${ }^{4}$, Carl T. D'angio ${ }^{5}$, \\ Abbot R. Laptook ${ }^{6}$, Pablo J. Sanchez ${ }^{7}$, Barbara J. Stoll ${ }^{8}$, Seetha Shankaran ${ }^{9}$, Krisa P. Van Meurs ${ }^{10}$, Noah Cook $^{11}$, \\ Rosemary D. Higgins ${ }^{12}$, Abhik Das ${ }^{3}$, Nancy S. Newman ${ }^{1}$, Kurt Schibler ${ }^{13}$, Barbara Schmidt ${ }^{11}$, C. Michael Cotten ${ }^{14}$, \\ Brenda B. Poindexter ${ }^{13,15}$, Kristi L. Watterberg ${ }^{16}$, William E. Truog ${ }^{17}$ and for the Eunice Kennedy Shriver National Institute \\ of Child Health and Human Development Neonatal Research Network
}

BACKGROUND: Extremely preterm infants (EPT, $<29$ weeks' gestation) represent only $0.9 \%$ of births in the United States; yet these infants are the focus of most published research. Moderately preterm neonates (MPT, 29-33 ${ }^{6 / 7}$ weeks) are an understudied group of high-risk infants.

METHODS: To determine the neonatal outcomes of MPT infants across the gestational age spectrum, and to compare these with EPT infants. A prospective observational cohort was formed in 18 level 3-4 neonatal intensive care units (NICUs) in the Eunice Kennedy Shriver NICHD Neonatal Research Network. Participants included all MPT infants admitted to NICUs and all EPT infants born at sites between January 2012 and November 2013. Antenatal characteristics and neonatal morbidities were abstracted from records using pre-specified definitions by trained neonatal research nurses.

RESULTS: MPT infants experienced morbidities similar to, although at lower rates than, those of EPT infants. The main cause of mortality was congenital malformation, accounting for $43 \%$ of deaths. Central Nervous System injury occurred, including intraventricular hemorrhage. Most MPT infants required respiratory support, but sequelae such as bronchopulmonary dysplasia were rare. The primary contributors to hospitalization beyond 36 weeks' gestation were inability to achieve adequate oral intake and persistent apnea.

CONCLUSIONS: MPT infants experience morbidity and prolonged hospitalization. Such morbidity deserves focused research to improve therapeutic and prevention strategies.
$\mathbf{E}$ xtremely preterm infants (EPT, $<29$ weeks' gestation) represent only $0.9 \%$ of births in the United States; yet these infants are the subject of the majority of published research on newborns $(1,2)$. Moderately preterm neonates (MPT, $29-33^{6 / 7}$ weeks), which constituted $2.8 \%$ of all births in the United States in 2013 and 22\% of all preterm births, are an understudied group of high-risk infants (1). A medline search using the terms moderately preterm, preterm, and randomized trial found only one randomized trial in MPT neonates-a surfactant trial in 1993 (3). Thus, the care of MPT infants is extrapolated from studies of EPT or full-term infants rather than from randomized trials specific to MPT infants. MPT infants are at risk for substantial short-term morbidity. Most published studies have focused on latepreterm infants (34-36 weeks' gestational age) and some have included a subset of MPT infants. These limited studies have shown higher rates of abnormal respiratory outcomes, cognitive functioning, school performance, and behavior (4-8). Because of their large numbers ( 93000 in 2013), morbidities experienced by MPT neonates represent a substantial public health burden $(7,8)$. Additional information about the morbidity in this group is needed from a contemporary cohort to inform the design of future interventional trials and improve the effectiveness of care. Thus, we performed a large prospective observational study of the outcomes in MPT infants and evaluated those outcomes by gestational age. In addition, we compared outcomes of MPT infants with those of EPT infants enrolled in a longstanding registry of the NICHD Neonatal Research Network (NRN).

\footnotetext{
${ }^{1}$ Department of Pediatrics, Rainbow Babies \& Children's Hospital, Case Western Reserve University, Cleveland, Ohio; ${ }^{2}$ Department of Pediatrics, University of lowa, lowa City, lowa; ${ }^{3}$ Social, Statistical and Environmental Sciences Unit, RTI International, Research Triangle Park, North Carolina; ${ }^{4}$ Division of Neonatology, University of Alabama at Birmingham, Birmingham, Alabama; ${ }^{5}$ University of Rochester School of Medicine and Dentistry, Rochester, New York; ${ }^{6}$ Department of Pediatrics, Women \& Infants Hospital, Brown University, Providence, Rhode Island; ${ }^{7}$ Department of Pediatrics, Nationwide Children's Hospital, Columbus, Ohio; ${ }^{8}$ Department of Pediatrics, Emory University School of Medicine, Children's Healthcare of Atlanta, Atlanta, Georgia; ${ }^{9}$ Department of Pediatrics, Wayne State University, Detroit, Michigan; ${ }^{10}$ Division of Neonatal and Developmental Medicine, Department of Pediatrics, Stanford University School of Medicine and Lucile Packard Children's Hospital, Palo Alto, California; ${ }^{11}$ Department of Pediatrics, The Children's Hospital of Philadelphia and The University of Pennsylvania, Philadelphia, Pennsylvania; ${ }^{12}$ Eunice Kennedy Shriver National Institute of Child Health and Human Development, National Institutes of Health, Bethesda, Maryland; ${ }^{13}$ Perinatal Institute, Cincinnati Children's Hospital Medical Center, Cincinnati, Ohio; ${ }^{14}$ Department of Pediatrics, Duke University, Durham, North Carolina; ${ }^{15}$ Department of Pediatrics, Indiana University School of Medicine, Indianapolis, Indiana; ${ }^{16}$ University of New Mexico Health Sciences Center, Albuquerque, New Mexico; ${ }^{17}$ Department of Pediatrics, Children's Mercy Hospital, Kansas City, Missouri. Correspondence: Michele C. Walsh (mcw3@case.edu) 


\section{Articles | Walsh et al.}

\section{METHODS}

The NRN is a network of neonatal intensive care units at 18 academic centers in the United States and a data coordinating center, which was formed to conduct research to improve the care of highrisk infants. All infants between $29^{0 / 7}$ and $33^{6 / 7}$ weeks' gestational age (GA) born between January 2012 and November 2013 and admitted within the first $72 \mathrm{~h}$ of life were included in the Moderately Preterm Registry. The comparison group comprised infants enrolled in the NRN Registry of inborn infants of gestational age $22^{0 / 7}$ to $27^{6 / 7}$ weeks (EPT) or having birth weight 401-1,000 g who were born during the same months. Infants who were born alive but who died in the delivery room were also included. Trained research nurses using prespecified definitions abstracted maternal demographic, pregnancy, delivery, and infant data from birth to discharge, transfer, death, or 40 weeks' postmenstrual age (PMA), whichever occurred first. Detailed information on the EPT cohort by week of GA was published recently in JAMA (9). The Institutional Review Board at each center approved the registries.

Neonatal information included birth weight (BW), GA, gender, race/ethnicity, mode of delivery, delivery room interventions, final status, and cause of death. GA was determined as the best obstetric estimate by using ultrasonography and/or the date of the last menstrual period. Neonatal morbidities were recorded for infants surviving for $>12 \mathrm{~h}$, and included respiratory distress syndrome, patent ductus arteriosus (PDA), modified Bell's Stage $\geqslant$ IIA necrotizing enterocolitis (NEC) (10), any intracranial hemorrhage according to the criteria of Papile (11), severe intracranial hemorrhage (grade III or IV), periventricular leukomalacia, retinopathy of prematurity (ROP) (12), bronchopulmonary dysplasia (BPD) defined as supplemental oxygen at 36 weeks' PMA, and earlyand late-onset sepsis defined by positive blood cultures before or after $72 \mathrm{~h}$ of age. Cranial sonograms and ROP exams were performed on the basis of usual center practice. Outcomes collected at 36 weeks' PMA included the reason for continued hospitalization (continued respiratory support; persistent apnea and/or bradycardia-defined by local center practice; inadequate oral feedings defined by continued gavage feedings; other). Data collection continued until discharge or 40 weeks' PMA, whichever occurred first. Only $16 \%$ of the cohort remained hospitalized at 40 weeks' PMA.

The NRN has maintained a registry of the characteristics and outcomes of EPT infants for over 20 years (9). We utilized this longstanding registry to compare a cohort of MPT infants with a cohort of inborn EPT infants born during the same months. We further analyzed the outcomes in MPT infants across the GA continuum. Statistical significance in this observational study was tested using the $\chi^{2}$-test for categorical variables and the Kruskall-Wallis test for continuous variables. Comparisons were made by GA and contrasted with the frequency seen in EPT infants born during the same period.

\section{RESULTS}

\section{Population and Pregnancy Characteristics}

Seven thousand and fifty-seven MPT infants met the study criteria. Of them, 6,419 (91\%) were inborn and 638 (9\%) were outborn. During the same period, 4,310 EPT infants were born at NRN sites. Maternal and infant characteristics are shown in Table 1. The average maternal age was $28.4 \pm 6.5$ (mean $\pm \mathrm{SD}$ ) years. Most women were White $(57 \%)$ and nonHispanic (83.3\%). Nearly half had completed high school and additional higher education (49.9\%). Almost all attended at least one prenatal visit (97\%). The majority of women were publicly insured (55.2\%), with a small percentage being uninsured (3.4\%).

Multiple births were common in our MPT cohort ( $n=2,059,29.2 \%)$. Frequently observed pregnancy complications included hypertension $(n=3,172,44.9 \%)$ and insulin- dependent diabetes $(n=527,7.5 \%)$. Prolonged preterm rupture of the membranes occurred in 1,267 (19.2\%), and clinical chorioamnionitis was noted in 498 (7.1\%). Overall, $85.3 \%$ of the cohort received antenatal steroids. Most mothers $(68.5 \%)$ received antibiotics in the $72 \mathrm{~h}$ prior to delivery, and $53.7 \%$ received magnesium sulfate. Four thousand four hundred and sixty-five (63.3\%) infants were delivered by cesarean section. The maternal characteristics of the MPT and EPT cohorts were similar (Table 1). Notable exceptions are a higher rate of Black race and lower rates of private insurance in the EPT cohort. EPT infants were more likely to receive resuscitation in the delivery room and higher rates of all interventions, including chest compressions.

\section{MORBIDITY}

\section{Cardiorespiratory Outcomes}

Respiratory illness was the most common morbidity noted (Table 2). With each 1-week increase in gestational age, surfactant administration declined by $\sim 10 \%$. Fifty percent of infants born at 29 weeks received surfactant compared with only $12.8 \%$ of those born at 33 weeks. In contrast, $77.8 \%$ of EPT infants were treated with surfactant. At 28 days of life, $59.7 \%$ of infants born at 29 weeks no longer required any respiratory support; at 33 weeks, $95.4 \%$ were without respiratory support. In comparison, only $15 \%$ of EPT infants were off all respiratory support by 28 days of life. Bronchopulmonary dysplasia (based on oxygen use at 28 days of life) occurred in $16 \%$ of MPT infants and in $50 \%$ of EPT infants. Patent ductus arteriosus (PDA) was diagnosed in only 754 MPT infants (10.7\%) compared with $43 \%$ of EPT infants. Most MPT infants diagnosed with PDA were not treated, but 150 (19.9\% with PDA) were treated with indomethacin or ibuprofen and 34 (4.5\% with PDA) with surgical ligation. In contrast, $60 \%$ of the EPT infants in whom PDA was diagnosed were treated.

\section{Central Nervous System Outcomes}

Overall, 58\% of MPT infants were screened with cranial ultrasound, compared with $97 \%$ of EPT infants. The frequency with which an ultrasound was performed increased with decreasing gestational age. An intracranial hemorrhage (ICH) was diagnosed in $13.3 \%$ of the MPT cohort and the overall percentage did not vary across the gestational ages evaluated. However, with decreasing gestational age the severity of ICH increased; $17.7 \%$ of 29 -week-old MPT infants who had undergone a head ultrasound had a grade 3 or 4 $\mathrm{ICH}$, whereas only $1.8 \%$ of 33 -week-old infants screened had a grade 3 or $4 \mathrm{ICH}$. In contrast to the MPT cohort, $26.9 \%$ of EPT infants had an ICH. Almost $30 \%$ of MPT infants received a late imaging study, with $26.5 \%$ of these showing an abnormal finding, including ventriculomegaly, periventricular leukomalacia, and porencephalic cyst. Among MPT infants, the frequency of these findings did not vary by gestational age.

Retinopathy of prematurity of any degree was diagnosed in $8 \%$ of the MPT cohort compared with $54 \%$ of the EPT cohort. Severe retinopathy (defined as stage 3 or worse) was 
Table 1. Population characteristics of moderately preterm and extremely preterm infants

\begin{tabular}{|c|c|c|c|}
\hline Characteristics & All MPT, $N=7,057(\%)$ & EPT, $N=4,310(\%)$ & $P$ value \\
\hline \multicolumn{4}{|l|}{ Maternal information } \\
\hline Maternal age (years; mean \pm SD) & $28.4 \pm 6.5$ & $28.1 \pm 6.3$ & 0.038 \\
\hline \multicolumn{4}{|l|}{ Highest level of education ${ }^{\mathrm{b}}$} \\
\hline High school or less & $2,522(50.1)$ & $1,611(50.4)$ & 0.739 \\
\hline \multicolumn{4}{|l|}{ Insurance status } \\
\hline Public or uninsured & $4,128(58.6)$ & $2,607(60.5)$ & 0.019 \\
\hline Private & $2,821(40.0)$ & $1,627(37.8)$ & \\
\hline Other/unknown & $101(1.4)$ & $74(1.7)$ & \\
\hline Unknown & $171(2.4)$ & $50(1.2)$ & \\
\hline \multicolumn{4}{|l|}{ Mother's race } \\
\hline Black & $2,232(31.6)$ & $1,703(39.5)$ & $<0.0001$ \\
\hline White & $4,025(57.0)$ & $2,163(50.2)$ & \\
\hline Other & $463(6.6)$ & $279(6.5)$ & \\
\hline Unknown & $337(4.8)$ & $165(3.8)$ & \\
\hline \multicolumn{4}{|l|}{ Pregnancy complications } \\
\hline Multiple birth & $2,059(29.2)$ & $1,193(27.7)$ & 0.087 \\
\hline Prenatal care & $6,834(97.0)$ & $4,140(96.1)$ & 0.012 \\
\hline Antibiotics given in this admission ${ }^{\mathrm{b}}$ & $4,951(71.6)$ & $3,087(72.0)$ & 0.650 \\
\hline Magnesium given ${ }^{\mathrm{b}}$ & $3,708(53.7)$ & $3,011(70.4)$ & $<0.0001$ \\
\hline \multicolumn{4}{|l|}{ Delivery mode } \\
\hline Vaginal & $2,582(36.6)$ & 1,449 (33.6) & $<0.0001$ \\
\hline Cesarean section & $4,465(63.3)$ & $2,855(66.3)$ & \\
\hline Unknown & $8(0.1)$ & $5(0.1)$ & \\
\hline \multicolumn{4}{|l|}{ Neonatal information } \\
\hline Birth weight, $\mathrm{kg}$ (mean $\pm \mathrm{SD})$ & $1,695 \pm 422$ & $839.0 \pm 251.6$ & $<0.0001$ \\
\hline Male $(n, \%)$ & $3,682(52.2)$ & $2,181(50.7)$ & 0.108 \\
\hline \multicolumn{4}{|l|}{ Resuscitation at delivery } \\
\hline Oxygen and/or bag/mask & $5,063(71.8)$ & $3,948(91.7)$ & $<0.0001$ \\
\hline Ventilation (CPAP or intubation) & $4,292(60.9)$ & $3,809(88.5)$ & $<0.0001$ \\
\hline Chest compressions & $180(2.6)$ & $338(7.9)$ & $<0.0001$ \\
\hline
\end{tabular}

aPercentages calculated on the basis of non-missing information.

${ }^{b}$ More than $10 \%$ of data are missing for: maternal education ( $n=2,023$ for MPT and $n=1,115$ for Generic Database (GDB)), clinical chorioamnionitis ( $n=73$ for MPT and $n=14$ for GDB), PROM ( $n=450$ for MPT and $n=353$ for GDB), antibiotics given in this admission ( $n=140$ for MPT and $n=21$ for GDB), antibiotics given at $72 \mathrm{~h}$ prior to delivery ( $n=155$ for MPT and $n=23$ for GDB), magnesium ( $n=155$ for MPT and $n=31$ for GDB), thermal wrap used ( $n=1,071$ for MPT and $n=433$ for GDB), temperature at admission ( $n=768$ for MPT and $n=637$ for GDB). 
Table 2. Neonatal outcomes for moderately preterm infants by gestational age and compared with extremely preterm infants

\begin{tabular}{|c|c|c|c|c|c|c|c|c|}
\hline & \multicolumn{2}{|c|}{ Gestational age } & \multirow[b]{2}{*}{$31, N=1,255(\%)$} & \multirow[b]{2}{*}{$32, N=1,712(\%)$} & \multirow[b]{2}{*}{$33, N=2,209(\%)$} & \multirow[b]{2}{*}{ All MPT, $N=7,057(\%)$} & \multirow[b]{2}{*}{ EPT, $N=4,310(\%)$} & \multirow[b]{2}{*}{$P$-value } \\
\hline & $29, N=815(\%)$ & $30, N=1,066(\%)$ & & & & & & \\
\hline Cardiorespiratory & $410(50.3)$ & $414(38.8)$ & $376(30.0)$ & $351(20.5)$ & $282(12.8)$ & $1,833(26.0)$ & 3,041 (77.8) & $\begin{array}{l}<0.0001 \\
*<0.0001\end{array}$ \\
\hline \multirow[t]{2}{*}{ Surfactant } & 778 (95.5) & 1,032 (96.8) & $1,230(98.0)$ & $1,673(97.7)$ & $2,156(97.6)$ & $6,869(97.3)$ & $3,523(81.7)$ & $\begin{array}{ll} & 0.0031 \\
* & <0.0001\end{array}$ \\
\hline & & & & & & & & $\begin{array}{l}<0.0001 \\
*<0.0001\end{array}$ \\
\hline $\begin{array}{l}\text { Respiratory support at } 28 \text { days among } \\
\text { those alive }\end{array}$ & $456(59.7)$ & $740(73.5)$ & $1,023(85.5)$ & $1,469(92.5)$ & 1,932 (95.4) & $5,620(85.4)$ & $549(15.7)$ & \\
\hline None & $35(4.58)$ & 34 (3.38) & $24(2.01)$ & $25(1.57)$ & $16(0.79)$ & $134(2.04)$ & $1,329(38.1)$ & \\
\hline Ventilator & $42(5.50)$ & $28(2.78)$ & $6(0.50)$ & $6(0.38)$ & $4(0.20)$ & $86(1.31)$ & $690(19.8)$ & \\
\hline CPAP & $231(30.2)$ & $205(20.4)$ & $144(12.0)$ & $88(5.54)$ & $73(3.60)$ & 741 (11.3) & $921(26.4)$ & \\
\hline \multirow[t]{2}{*}{ Oxygen only } & $160(19.7)$ & $167(15.7)$ & $148(11.8)$ & $133(7.78)$ & $146(6.62)$ & 754 (10.7) & $1,637(41.9)$ & $\begin{array}{l}<0.0001 \\
*<0.0001\end{array}$ \\
\hline & & & & & & & & $\begin{array}{ll} & 0.0005 \\
* & <0.0001\end{array}$ \\
\hline PDA treatment in those with PDA diagnosis & $100(62.5)$ & $114(68.7)$ & $113(76.4)$ & $113(85.0)$ & $126(86.3)$ & $566(75.2)$ & $636(38.9)$ & \\
\hline None & $50(31.3)$ & $48(28.9)$ & $25(16.9)$ & $16(12.0)$ & $11(7.53)$ & $150(19.9)$ & $696(42.5)$ & \\
\hline Medical & $10(6.25)$ & $4(2.41)$ & $8(5.41)$ & $3(2.26)$ & $9(6.16)$ & $34(4.52)$ & $92(5.6)$ & \\
\hline Surgery & $0(0)$ & $0(0)$ & $2(1.35)$ & $1(0.75)$ & $0(0)$ & $3(0.40)$ & $213(13.0)$ & \\
\hline \multicolumn{9}{|l|}{ Both medical and surgical } \\
\hline \multicolumn{9}{|l|}{ Central nervous system } \\
\hline Head ultrasound first 28 days $(n, \%)^{a}$ & $769(94.4)$ & $919(86.5)$ & $952(76.2)$ & $821(48.9)$ & $607(28.2)$ & $4,068(58.4)$ & $3,798(97.14)$ & $\begin{array}{l}<0.0001 \\
{ }^{*}<0.0001\end{array}$ \\
\hline Any grade intracranial hemorrhage & $113(14.8)$ & $130(14.3)$ & $114(12.1)$ & $98(12.0)$ & $82(13.7)$ & $537(13.3)$ & $995(26.93)$ & $\begin{array}{ll} & 0.3250 \\
* & <0.0001\end{array}$ \\
\hline Max grade intracranial hemorrhage & & & & & & & & $\begin{array}{ll} & 0.0047 \\
* & <0.0001\end{array}$ \\
\hline Grade 1 & $72(63.7)$ & $85(65.4)$ & $82(71.9)$ & $85(86.7)$ & $58(70.7)$ & $382(71.1)$ & $334(33.6)$ & \\
\hline Grade 2 & $21(18.6)$ & $30(23.1)$ & $16(14.0)$ & $5(5.10)$ & $13(15.9)$ & $85(15.8)$ & 195 (19.6) & \\
\hline Grade 3 & $8(7.08)$ & $11(8.46)$ & $4(3.51)$ & $4(4.08)$ & $5(6.10)$ & $32(5.96)$ & $210(21.1)$ & \\
\hline Grade 4 & $12(10.6)$ & $4(3.08)$ & $12(10.5)$ & $4(4.08)$ & $6(7.32)$ & $38(7.08)$ & $256(25.7)$ & \\
\hline Late imaging done & $565(70.8)$ & $616(59.4)$ & $501(40.8)$ & $233(14.2)$ & $109(5.21)$ & $2,024(29.8)$ & $3,137(80.39)$ & $\begin{array}{l}<0.0001 \\
*<0.0001\end{array}$ \\
\hline Late image Abnormal & $127(22.5)$ & $151(24.5)$ & $133(26.5)$ & $78(33.6)$ & $48(44.0)$ & $537(26.5)$ & $828(26.40)$ & $\begin{array}{l}<0.0001 \\
{ }^{*} 0.9103\end{array}$ \\
\hline
\end{tabular}


Gestational age

$29, N=815(\%) \quad 30, N=1,066(\%) \quad 31, N=1,255(\%) \quad 32, N=1,712(\%) \quad 33, N=2,209(\%) \quad$ All MPT, $N=7,057$ (\%) $\quad$ EPT, $N=4,310(\%) \quad P$-value

$\begin{array}{lllllll}691(84.8) & 770(72.2) & 521(41.5) & 299(17.5) & 116(5.25) & 2,397(34.0) & 3,364(86.04)\end{array}$

Exam for ROP

$2(0.29)$

$1(0.13)$

1 (0.19)

$0(0)$

$0(0)$

$4(0.17)$

$372(11.06)$

$*<0.0001$

ROP stage 3 or greater

$10.3 \pm 5.3$

$10.0 \pm 4.5$

$9.6 \pm 4.3$

$9.9 \pm 10.4$

$9.6 \pm 13.0$

$9.8 \pm 9.3$

$\begin{array}{lcccc}731(90.0) & 952(89.6) & 1,065(85.1) & 1,381(80.9) & 1,784(80.9) \\ 28.5 \pm 12.1 & 22.5 \pm 15.7 & 15.5 \pm 10.3 & 9.7 \pm 14.7 & 5.9 \pm 15.7 \\ 45.6 \pm 13.2 & 38.0 \pm 12.5 & 29.4 \pm 12.3 & 22.2 \pm 16.7 & 15.1 \pm 14.7 \\ 37(4.6) & 40(3.8) & 28(2.2) & 33(1.9) & 31(1.4)\end{array}$

$5,913(84.0)$

First oral feed, day $(\text { mean } \pm S D)^{b}$

Full oral feed, day $(\text { mean } \pm S D)^{b}$

NEC, any

$37(4.6)$

40 (3.8)

$10(1.23)$

$$
17(1.60)
$$

$10(0.80)$

$6(0.35)$

$9(0.41)$

$52(0.74)$

$13.5 \pm 16.2$

$26.0 \pm 17.7$

169 (2.4)

$\begin{array}{cc}9.9 \pm 7.9 & <0.0001 \\ & { }^{*} 0.66 \\ 3,431(96.5 \%) & <0.0001 \\ & { }^{*}<0.0001 \\ \text { NA } & <0.0001 \\ \text { NA } & <0.0001 \\ 343(8.8 \%) & <0.0001 \\ & *<0.0001\end{array}$

Early-onset infection $(n, \%)^{\mathrm{a}}$

Organism $^{\mathrm{b}}$

\section{Group B Strep}

E. coli

Other Gram positive

Other Gram negative

Other

Infant received antibiotics for $\geqslant 5$ days

\section{Late-onset infection}

\section{Phototherapy given}

\section{Phototherapy Days (mean, IQR)}

Highest total serum bilirubin (mean, IQR)

$\begin{array}{ccccc}1(10.0) & 2(11.8) & 3(30.0) & 0(0) & 2(22.2) \\ 2(20.0) & 2(11.8) & 1(10.0) & 2(33.3) & 2(22.2) \\ 3(30.0) & 11(64.7) & 4(40.0) & 4(66.7) & 3(33.3) \\ 3(30.0) & 1(5.88) & 2(20.0) & 0(0) & 0(0) \\ 1(10.0) & 1(5.88) & 0(0) & 0(0) & 2(22.2) \\ 170(20.9) & 189(17.7) & 200(16.0) & 194(11.3) & 223(10.1) \\ & & & & \\ 67(8.26) & 49(4.62) & 39(3.13) & 31(1.82) & 35(1.59) \\ 749(91.9) & 959(90.0) & 1,070(85.3) & 1,359(79.4) & 1,515(68.7) \\ 5.0(3.0,6.0) & 4.9(3.0,6.0) & 4.4(2.0,6.0) & 4.0(2.0,5.0) & 3.8(2.0,5.0) \\ 8.3(6.8,9.7) & 8.7(7.3,10.0) & 9.1(7.7,10.4) & 9.6(8.1,11.2) & 9.9(8.3 .11 .6) \\ & & & & \end{array}$

Full oral feeds ( $n=1,025$ overall, 29 weeks $\mathrm{GA}=157,30$ weeks $\mathrm{GA}=182,31$ weeks $\mathrm{GA}=198,32$ weeks $\mathrm{GA}=241,33$ weeks $\mathrm{GA}=247)$. Date of first oral feed was not tracked in the EPT cohort.

aPercentages calculated on the basis of non-missing information. All categories have $<10 \%$ missing data. First $P$-value compares the MPT gestational age distribution. Second $P$-value with an ${ }^{*}$ compares all MPT vs. EPT. ${ }^{b}$ More than $10 \%$ of data are missing for: days to regain weight $(n=944$ overall, 29 weeks $G A=76,30$ weeks $G A=90,31$ weeks $G A=136,32$ weeks $G A=207,33$ week $G A=435)$. 


\section{Articles | Walsh et al.}

diagnosed in $11 \%$ of the EPT cohort, but in only four $(0.17 \%)$ infants in the MPT cohort-all had stage 3 disease and none needed treatment.

\section{Nutrition and Infectious Outcomes}

MPT infants took, on average, 10 days to regain their birth weight. Although this figure varied significantly across the gestational age spectrum, the small differences are unlikely to be clinically meaningful. EPT infants regained their birth weight in a similar time frame of 9.9 days. The first oral feed was offered to MPT infants at about 33 weeks' PMA, regardless of GA at birth, although the time to reach that benchmark varied by GA. Less mature infants took longer to achieve full enteral feeds (17 days at 29 weeks' GA) than did more mature infants ( 9 days at 33 weeks' GA). The vast majority of both MPT and EPT infants were fed human milk ( $84 \%$ vs. $99 \%$ ), with less mature MPT infants more likely to receive human milk than more mature MPT infants $(90 \%$ at 29 weeks vs. $80.9 \%$ at 33 weeks, $P<0.001$ ).

As expected, necrotizing enterocolitis was diagnosed less frequently in MPT infants than in EPT infants (2.4\% vs. $8 \%$ ), and was more likely to be treated with medical treatment only (1.7\% vs. $4 \%)$.

MPT infants experienced early-onset sepsis at similar rates to EPT $(0.7 \%$ vs. $1.8 \%)$ with a predominance of Gram-positive organisms, including Group B Streptococcus (33/52 (63\%) infections). No infants in either cohort were infected with Listeria. Late-onset infection occurred less frequently in MPT than in EPT infants (3.2\% vs. $19 \%)$.

\section{Mortality and Hospital Course}

Overall, $2.9 \%$ of the MPT cohort died compared with $24 \%$ of the EPT cohort. Deaths occurred early, with most MPT infants dying at 7-12 days of age. The primary cause of death was related to congenital malformation (43\%) in the MPT cohort and to respiratory distress syndrome in the EPT cohort (Table 2). Although EPT infants remained in the hospital for an average $99 \pm 46$ days and usually were hospitalized past their due date, MPT infants were discharged at an earlier postmenstrual age, with $38.1 \%$ discharged by 36 weeks' PMA. The reasons for MPT infants remaining in the hospital at 36 weeks included inadequate oral intake $(69.8 \%)$, apnea or bradycardia (37.5\%), and continued respiratory issues (16.7\%). By 40 weeks' PMA, $83.9 \%$ of MPT infants were discharged home with an average weight of $2.4 \mathrm{~kg}$, compared with $58 \%$ of EPT infants.

\section{DISCUSSION}

Our cohort study contributes valuable information that will inform both clinicians and parents about the expected outcomes of these infants. The vast majority of neonatal clinical trials have focused on EPT infants. Yet, MPT infants experience many of the same risks for adverse outcomes, albeit at lower rates than EPT infants. Given their much larger numbers in the population, MPT infants spend a substantial number of days in intensive care units and contribute significantly to the societal costs of neonatal intensive care and to the emotional toll on their families. The burden of disease borne by MPT infants is substantial. For example, based on estimates from our study and from the national birth data, 12,447 MPT and 10,907 EPT infants suffered an ICH (1). This suggests that from an absolute number perspective, the MPT cohort may contribute to the burden of disease more than does the EPT cohort.

Research that focuses on the large group of MPT infants may generate novel ideas to support optimal growth and development and reduce the duration of hospitalization. Conditions associated with developmental immaturity, including apnea and bradycardia and inadequate oral feeding, were the predominant causes of continued hospitalization at 36 weeks. Across the MPT infants' GA continuum, oral feedings were first introduced, on average, at 33 weeks' PMA. Approaches for assessing an infant's readiness for oral feeding vary (13). It is possible that a more standardized approach and earlier introduction of oral feeding may facilitate the development of this skill. Oral feeding of the MPT infant is an area ripe for additional investigation. There are also differences in the approach to treatment of apnea of prematurity, including when to discontinue treatment with methylxanthines. After discontinuation of methylxanthines, most neonatologists in the United States continue hospitalization for an apnea-free period of observation of variable duration $(14,15)$. It is not known whether being discharged on methylxanthine treatment is a safe and effective method to reduce the length of stay.

An often-stated "rule of thumb" is that families may expect a preterm infant to be discharged home by their estimated due date. However, among MPT infants, 38\% were discharged home by 36 weeks' PMA, 4 weeks prior to their estimated due date, and $43 \%$ were discharged home by 40 weeks. Infants with congenital malformation were overrepresented in the population remaining in hospital at 40 weeks' PMA.

Our study can be compared with an analysis of preterm morbidity led by Manuck and conducted in the NICHD Maternal Fetal Network as a study nested within a large observational study to develop quality measures for intrapartum obstetric care (Assessment of Perinatal Excellence; APEX) $(16,17)$. Mothers who were at at least 23 weeks of gestation and up to $35^{6 / 7}$ weeks of gestation with a live fetus on admission had their data abstracted from records during a 24 -h period of randomly selected days from 25 hospitals nationwide between 2008 and 2011; the main study included 115,502 women. In the nested study, infants with anomalies and multiple gestations were excluded, as were infants who died in the delivery room and those $>36$ weeks. Once all exclusions were applied, the original sample of 118,422 infants was reduced to 8,334 infants, of whom 1,877 were MPT. Thus, a subset of the APEX population included MPT infants of similar gestational age (29-33 weeks) as our cohort. The outcomes evaluated were reported as major morbidity, as defined by pulmonary hypertension, intraventricular hemorrhage of grade 3 or 4 , hypoxic-ischemic encephalopathy, 
necrotizing enterocolitis stage 2 or 3, or bronchopulmonary dysplasia. The rates of major morbidity increased with decreasing GA, which parallels our data. The rate of major morbidity was $22.5 \%$ at 29 weeks and decreased to $4.2 \%$ at 33 weeks. Our study includes a larger sample size and a more robust slate of outcome variables.

Another recent cohort study, The Late and Moderately Preterm Birth Study (LAMBS), was conducted by Boyle et al. (18) in the United Kingdom. This prospective, populationbased study included outcomes for infants born at 3236 weeks' gestation from a geographic area in Northern England in 2009-2010. The study included 1,376 infants, of whom 1,125 (99.5\%) were discharged home. This cohort overlaps with our study at 32 and 33 weeks' gestation $(n=132)$, but excluded infants with congenital anomalies. Their findings were similar to ours in that MPT infants had a gradient of increasing risk with decreasing gestation, including an increasing length of stay.

Our study has several strengths. The infants were delivered at 18 level 3-4 centers across the United States and include both inborn and outborn infants, which contributes to the generalizability of the information. Another strength is that all data were collected by experienced research staff using standardized definitions, which were identical between the MPT and EPT cohorts. The large cohort allows us to present results by each week of GA, providing more precise information for counseling. One limitation is that information on the reason for preterm delivery was not uniformly collected for either inborn or outborn infants. Another possible concern is that the NRN population may not reflect similar populations of MPT that are not transferred to level 3 centers. Ninety percent of the cohort in this population was born at the NRN center and only $10 \%$ was transferred after birth. We have previously compared the outcome of infants in NRN centers and in the Vermont Oxford Network of both academic and private practice level 2 and level 3 units, and found the infant population to be comparable (19). Thus, we believe that the findings in this study are generalizable to other US neonatal intensive care units.

\section{CONCLUSION}

About 100,000 MPT infants are born in the United States each year but remain an understudied group. Because of the high numbers of MPT births in the United States, morbidities in this group are an important public health concern. This study will inform the counseling of parents faced with the birth of a MPT infant. MPT infants experience significant morbidity, as evidenced by the high rates of respiratory support and prolonged hospitalization. Such morbidity deserves focused research with an aim to develop novel therapeutic and prevention approaches.

\section{ACKNOWLEDGMENTS}

Funding Sources: The National Institutes of Health, the Eunice Kennedy Shriver National Institute of Child Health and Human Development (NICHD), and the National Center for Advancing Translational Sciences provided grant support for the Neonatal Research Network's Moderately Preterm Registry through cooperative agreements. Although NICHD staff did have input into the study design, conduct, analysis, and manuscript drafting, the content is solely the responsibility of the authors and does not necessarily represent the official views of the National Institutes of Health.

\section{AUTHOR CONTRIBUTIONS}

The contributions made by each author for this manuscript are given in detail below.

The MPR Subcommittee investigators have monthly conference calls, during which protocol design and implementation issues are discussed, manuscripts are reviewed, and input obtained. The following authors have made significant contributions as determined by the Uniform Requirements for Manuscripts Submitted to Biomedical Journals:

Michele C. Walsh, MD, MS, is the Lead Principal Investigator (PI) at Case Western Reserve University (CWRU) and the Chair of the Moderately Preterm Registry Protocol Subcommittee. She developed the study and managed protocol implementation. As the PI at CWRU, she oversaw enrollment at the site-which enrolled 302 infants in this study. Walsh drafted the manuscript and received input from the authors below as part of manuscript revision. Walsh had full access to all the data in the study and takes responsibility for the integrity of the data and the accuracy of the data analysis.

Edward F. Bell, MD, is the PI at the University of lowa and a member of the Moderately Preterm Registry Protocol Subcommittee. He helped develop the study and manage protocol implementation. As the Pl at the University of lowa, he oversaw enrollment at the site-which enrolled 495 infants in this study. He contributed critical revisions of the manuscript and approved the final manuscript for submission.

Sarah Kandefer, BS, served as the primary statistician for the study, and completed the statistical analyses for the paper. She developed the tables for the paper and provided critical revision to the manuscript and approved the final version of the manuscript.

Waldemar A. Carlo, MD, is the $\mathrm{Pl}$ at the University of Alabama at Birmingham (UAB) and a member of the Moderately Preterm Registry Protocol Subcommittee. He helped develop the study and manage protocol implementation. As the PI at UAB, he oversaw enrollment at the site-which enrolled 537 infants in this study. He contributed critical revisions of the manuscript and approved the final manuscript for submission.

Carl T. D'Angio, MD, is the PI at the University of Rochester and a member of the Moderately Preterm Registry Protocol Subcommittee. He helped develop the study and manage protocol implementation. As the PI at the University of Rochester, he oversaw enrollment at the site-which enrolled 617 infants in this study. He contributed critical revisions of the manuscript and approved the final manuscript for submission.

Abbot R. Laptook, MD, is the PI at Brown University and a member of the Moderately Preterm Registry Protocol Subcommittee. He helped develop the study and manage protocol implementation. As the PI at Brown University, he oversaw enrollment at the site-which enrolled 424 infants in this study. He contributed critical revisions of the manuscript and approved the final manuscript for submission.

Pablo J. Sanchez, MD, is the PI at Nationwide Children's Hospital and a member of the Moderately Preterm Registry Protocol Subcommittee. He helped develop the study and manage protocol implementation. As the PI at Nationwide Children's Hospital, he oversaw enrollment at the sitewhich enrolled 512 infants in this study. He contributed critical revisions of the manuscript and approved the final manuscript for submission.

Barbara J. Stoll, MD, is the PI at Emory University and the Vice Chair of the Moderately Preterm Registry Protocol Subcommittee. She helped develop the study and manage protocol implementation. As the PI at Emory University, she oversaw enrollment at the site-which enrolled 335 infants in this study. She contributed critical revisions of the manuscript and approved the final manuscript for submission.

Seetha Shankaran, MD, is the PI at Wayne State University and a member of the Moderately Preterm Registry Protocol Subcommittee. She helped develop the study and manage protocol implementation. As the PI at Wayne State University, she oversaw enrollment at the site-which 
enrolled 363 infants in this study. She contributed critical revisions of the manuscript and approved the final manuscript for submission.

Krisa P. Van Meurs, MD, is the PI at Stanford University and a member of the Moderately Preterm Registry Protocol Subcommittee. She helped develop the study and manage protocol implementation. As the PI at Stanford University, she oversaw enrollment at the site-which enrolled 205 infants in this study. She contributed critical revisions of the manuscript and approved the final manuscript for submission.

Betty R. Vohr, MD, is the Follow-up PI at Brown University and a member of the Moderately Preterm Registry Protocol Subcommittee. She helped develop and implement the study and contributed critical revisions of the manuscript and approved the final manuscript for submission.

Rosemary D. Higgins, MD, served as the Program Scientist for the NICHD NRN and is a member of the Moderately Preterm Registry Protocol Subcommittee. Dr Higgins helped develop the protocol, oversaw recruitment, and assisted with data edits from the sites. She also provided critical revision to the manuscript and approved the final version of the manuscript.

Abhik Das, PhD, is the PI for the NRN Data Coordinating Center and a member of the Moderately Preterm Registry Protocol Subcommittee. Das oversaw all aspects of the statistical analysis, provided critical revisions to the manuscript, and approved the final version of the manuscript.

Ellen C. Hale, BS, RN, CCRC, is the Coordinator at Emory University and a member of the Moderately Preterm Registry Protocol Subcommittee. She helped develop the study and manage protocol implementation. As the Coordinator at Emory University, she enrolled 335 infants in this study. She contributed critical revisions of the manuscript and approved the final manuscript for submission.

Nancy S. Newman, RN, is the Coordinator at CWRU and a member of the Moderately Preterm Registry Protocol Subcommittee. She helped develop the study and manage protocol implementation. As the Coordinator at CWRU, she enrolled 302 infants in this study. She contributed critical revisions of the manuscript and approved the final manuscript for submission.

Kurt Schibler, MD, is the PI at Cincinnati Children's Medical Center (CCMC). As the PI he oversaw enrollment at the site-which enrolled 683 infants in this study. He contributed critical revisions of the manuscript and approved the final manuscript for submission.

Barbara Schmidt, MD, is the PI at the University of Pennsylvania. As the PI she oversaw enrollment at the site-which enrolled 511 infants in this study. She contributed critical revisions of the manuscript and approved the final manuscript for submission.

C. Michael Cotten, MD, MHS, is the PI at Duke University. As the PI he oversaw enrollment at the site-which enrolled 457 infants in this study. He contributed critical revisions of the manuscript and approved the final manuscript for submission.

Kathleen A. Kennedy, MD, MPH, is the PI at the University of Texas Health Science Center at Houston. As the PI she oversaw enrollment at the sitewhich enrolled 456 infants in this study. She contributed critical revisions of the manuscript and approved the final manuscript for submission.

Brenda B. Poindexter, MD, MS, is the PI at Indiana University. As the PI, she oversaw enrollment at the site-which enrolled 333 infants in this study. She contributed critical revisions of the manuscript and approved the final manuscript for submission.

Kristi L. Watterberg, MD, is the PI at the University of New Mexico. As the $\mathrm{PI}$, she oversaw enrollment at the site-which enrolled 242 infants in this study. She contributed critical revisions of the manuscript and approved the final manuscript for submission.

William E. Truog, MD, is the PI at Children's Mercy Hospital, Kansas City. As the $\mathrm{Pl}$, he oversaw enrollment at the site-which enrolled 172 infants in this study. He contributed critical revisions of the manuscript and approved the final manuscript for submission.
Presentations: portions of the material in this manuscript were presented at the Pediatric Academic Society Meeting, May 2014, Vancouver, BC, Canada.

Disclosure: The authors declare no conflict of interest.

\section{REFERENCES}

1. Martin JA, Hamilton BE, Osterman MJ, Curin SC, Mathews TJ. Births: final data for 2013. Natl Vital Stat Rep 2015;64:1-65.

2. NICHD Neonatal Research Network, protocol list. neonatal.rti.org. Accessed 11 March 2016.

3. Kattwinkel J, Bloom BT, Delmore P, et al. Prophylactic administration of calf lung surfactant is more effective than early treatment of respiratory distress syndrome in neonates of 29 through 32 weeks gestation. Pediatrics 1993;92:90-8.

4. Been JV, Lugtenberg MJ, Smets E, et al. Preterm birth and childhood wheezing disorders: a systematic review and meta-analysis. PLoS Med 2014;11:e1001596.

5. Colin AA, McEvoy C, Castile RG. Respiratory morbidity and lung function in preterm infants of 32 to 36 weeks gestational age. Pediatrics 2010;126:115-8.

6. Saarenpaa HK, Tikanmaki M, Siola-Leppanen M, et al. Lung function in very low birth weight adults. Pediatrics 2015;136:642-50.

7. Lindstrom K, Winbladh B, Haglund B, Hiern A. Preterm infants as young adults: a Swedish national cohort study. Pediatrics 2007;120:70-7.

8. Moster D, Lie RT, Markestad T. Long term medical and social consequences of preterm birth. NEJM 2008;359:262-73.

9. Stoll BJ, Hansen NI, Bell EF, et al. Trends in care practices, morbidity and mortality of extremely preterm neonates, 1993-2012. JAMA 2015;314: 1039-51.

10. Walsh MC, Kliegman RM. Necrotizing enterocolitis: treatment based on staging criteria. Pediatr Clin North Am 1986;33:179-201.

11. Papille LA, Burstein J, Burstein R, Koffler H. Incidence and evolution of supependymal and intraventricular hemorrhage: a study of infants with birth weights less than 1,500 gm. J Pediatr 1978;92:529-34.

12. International Committee for the Classification of Retinopathy of Prematurity. The international classification of retinopathy of prematurity revisited. Arch Ophthalmol 2005;123:991-9.

13. Thoyre SM, Shaker CS, Pridham KF. The early feeding skills assessment for preterm infants. Neonatal Netw 2005;24:7-16.

14. Eichenwald EC, Zupancic JA, Mao WY, Richardson DK, McCormick MC, Escobar GJ. Variation in diagnosis of apnea in moderatelyly preterm infants predicts length of stay. Pediatrics 2011;127:e53-8.

15. Eichenwald ECthe Committee on the Fetus and Newborn, American Academy of Pediatrics. Apnea of prematurity. Pediatrics 2015;137: e20153757.

16. Manuck TA, Murguia Rice M, Bailit JL, et al. Preterm neonatal morbidity and mortality by gestational age: a contemporary cohort. Am J Obstet Gynecol 2016;215:103.e1-14.

17. Grobham WA, Bailit JL, Rice MM, et al. Can differences in obstetric outcomes be explained by differences in the care provided? The MFMU APEX Study. Am J Obstet Gynecol 2014;211:147.e1-16.

18. Boyle EM, Johnson S, Manktelow B, et al. Neonatal outcomes and delivery of care for infants born late preterm or moderatelyly preterm: a prospective population based study. Arch Dis Child Fetal Neonatal Ed 2015;100:F479-85.

19. Walsh MC, Yao Q, Horbar JD, Carpenter JH, Lee SK, Ohlsson A. Changes in the use of postnatal steroids for bronchopulmonary dysplasia in 3 large neonatal networks. Pediatrics 2006;118:e1328-35. 